\title{
Article
}

\section{Aggregation Behavior of the Styrene Units in Oil Gel of Styrene-Butadiene-Styrene Triblock Copolymer}

\author{
Shigeru MACHIDA, Naomi TAKAHASHI, Yasuhiro MATSUDA, and Shigeru TASAKA \\ Department of Materials Science and Chemical Engineering, Faculty of Engineering, Shizuoka University, \\ 3-5-1 Johoku, Naka-ku, Hamamatsu 432-8561, Japan \\ (Received : June 13, 2008)
}

\begin{abstract}
Differential scanning calorimetry (DSC) and viscoelastic measurements were carried out for oil gels formed by styrene-butadiene-styrene triblock copolymer (SBS) and naphten oil or 1-octadecene to investigate the mechanism of their gelation. There were three endothermic peaks in the DSC curves assigned as the glass transition of the butadiene units, that of the styrene units, and the dissolution enthalpy of the styrene units and solvent oils. The $G^{\prime}$ of the SBS gels showed a specific depression near the glass transition temperature of the styrene units, determined by DSC. The modulus curve had also a shoulder at the gel melting temperature determined by the tilting test tube method. The structure and gelation mechanism of SBS gel were discussed on the results of the measurements.
\end{abstract}

Key Words: Styrene-butadiene-styrene triblock copolymer / Naphten oil / Oil gel / Differential scanning calorimetry

\section{INTRODUCTION}

Polymer gels are formed by three-dimensional network of polymer chains holding solvent molecules. Polymer chains in the network are linked to each other by chemical bonds or physical interaction. Hydrogen bonds, ionic bonds, or coordinate bonds can form physical gels of poly(vinyl alcohol $)^{1)}$, poly(acrylic acid) $)^{2)}$, or poly(4-vinylpyridine) $)^{3)}$, respectively. Polymer chains have to form some structure to be linked by these physical bonds and the structure of polymer chains has been studied by various measuring techniques such as electron microscopy, atomic force microscopy, dynamic light scattering, and small angle X-ray and neutron scattering.

Styrene-butadiene-styrene triblock copolymer (SBS) which is one of the important elastomers ${ }^{46}$, is also known to form physical gels in mineral oils. ${ }^{7-9)}$ SBS oil gel is applied as constructing materials and shock absorber for its rubbery elasticity. The gel networks of SBS in oil is formed by the aggregative interaction of the styrene units in a selective solvents which is a good solvent for butadiene units, but a poor solvent for styrene unit, and seggregative interaction between the styrene and butadiene units. The aggregative and seggregative interactions of the block copolymer of styrene and butadiene were extensively studied by Kotaka and his colleagues. ${ }^{5,10-13)}$ They also studied the structure and rheological properties of the diblock and triblock copolymers of styrene and butadiene in selective solvents.
Watanabe et al. ${ }^{5)}$ investigated the structure and gelation mechanism of SBS gel formed in $n$-tetradecane by dynamic measurements. They introduced microphase-separated structure of styrene and butadiene units in SBS to explain the rheological properties of their SBS gel. The proposed structure of the gel is constructed by the quasinetwork of SBS formed by the framework of butadiene units crosslinked by the aggregative phase of styrene units. At high temperature, the chain transfer process of the styrene units from one aggregative phase to another plays an important role for the rheological properties.

In this manuscript, differential scanning calorimetry (DSC) was carried out for the SBS gel in naphten oil to investigate the thermal mechanism of the gelation. The glass transition temperature (Tg), and upper critical solution temperature (UCST) of the styrene units were determined for the SBS gels with various contents of SBS. The rheological properties of our SBS gel were also investigated by viscoelastic measurement and the tilting test tube method to determine the gel melting temperature.

\section{EXPERIMENTAL}

Styrene-butadiene-styrene triblock copolymer (SBS) used in this study was a commercial product of Asahi Kasei Chemicals Co. (T-420), supplied in the form of porous pellets. The weight fraction of styrene units is reported to be $30.0 \%$. 
The weight average molecular weight $\left(M_{\mathrm{w}}\right)$ of SBS and styrene unit is reported to be 110,000 (calibrated with standard polystyrene) and 15,000, respectively. Atactic polystyrene sample used for DSC measurements was purchased from Aldrich. $M_{\mathrm{w}}$ and number average molecular weight $M_{\mathrm{n}}$ of this polystyrene sample are 350,000 and 170,000, respectively.

Commercially available napthen oil was used as the solvent for SBS. The napthen oil was the product of Sankyo Yuka Kogyo Co. (SNH 220), and reported to contain 15 wt\% polar aromatics, 45 wt\% napthen aromatics, and 40 wt\% saturates. 1-Octadecene purchased from Tokyo Chemical Industry Co., Ltd was also used for DSC measurements to confirm the absence of selective adsorption of napthen oil to SBS.

SBS oil gels were prepared with a pitched-blade propeller mixer (Silverson, model L4R). Napthen oil melted in a stainless cup at $185^{\circ} \mathrm{C}$, was gradually added to preheated SBS. After SBS was dissolved into napthen oil to form transparent solution, it was allowed to stand at room temperature. Transparent gels were obtained for the mixture of which the SBS content was over $7 \mathrm{wt} \%$.

DSC measurements were conducted with Shimadzu DSC-60 under air atmosphere. The gel samples were first cooled with liquid nitrogen, then heated at $20{ }^{\circ} \mathrm{C} / \mathrm{min}$ from $-110^{\circ} \mathrm{C}$ to $150{ }^{\circ} \mathrm{C}$ to determine $\mathrm{Tg}$ of the butadiene and styrene units. DSC measurements from $30^{\circ} \mathrm{C}$ to $180^{\circ} \mathrm{C}$ were also carried out to estimate the heat of dissolution of the styrene units to napthen oil.

Viscoelastic measurements were carried out with Rheoplus MCR101 (Anton Paar Co.). The samples were heated at $5^{\circ} \mathrm{C} / \mathrm{min}$ from $-20^{\circ} \mathrm{C}$ to $200^{\circ} \mathrm{C}$. The measurement frequency was fixed to $1 \mathrm{~Hz}$.

The melting temperature of SBS was defined to be the temperature when SBS gels began to flow. SBS gels were first prepared in a test tube with the diameter of $16 \mathrm{~mm}$ by the same recipe mentioned above, and were heated again to measure their melting temperature.

\section{RESULTS}

Fig. 1a shows the DSC curves for SBS gels in naphten oil with different weight fraction of SBS. The DSC curves of naphten oil without SBS and bulk SBS indicated by $0 \mathrm{wt} \%$ and $100 \mathrm{wt} \%$, respectively are also illustrated in the figure. Although the data points in the DSC curves scattered, there were two endothermic peaks at ca. -70 and $50{ }^{\circ} \mathrm{C}$ in the all the curves of SBS. The endothermic transitions around $-70^{\circ} \mathrm{C}$ were assigned as the glass transition of the butadiene units. Fig. 1b, which is an enlarged figure of Fig 1a, also shows small endothermic transitions around $50^{\circ} \mathrm{C}$. As the Tg of bulk sample of the homopolymer of styrene is ca. $100^{\circ} \mathrm{C}$, this transition temperature was too low to be the glass transition of the styrene units which were completely immiscible with the
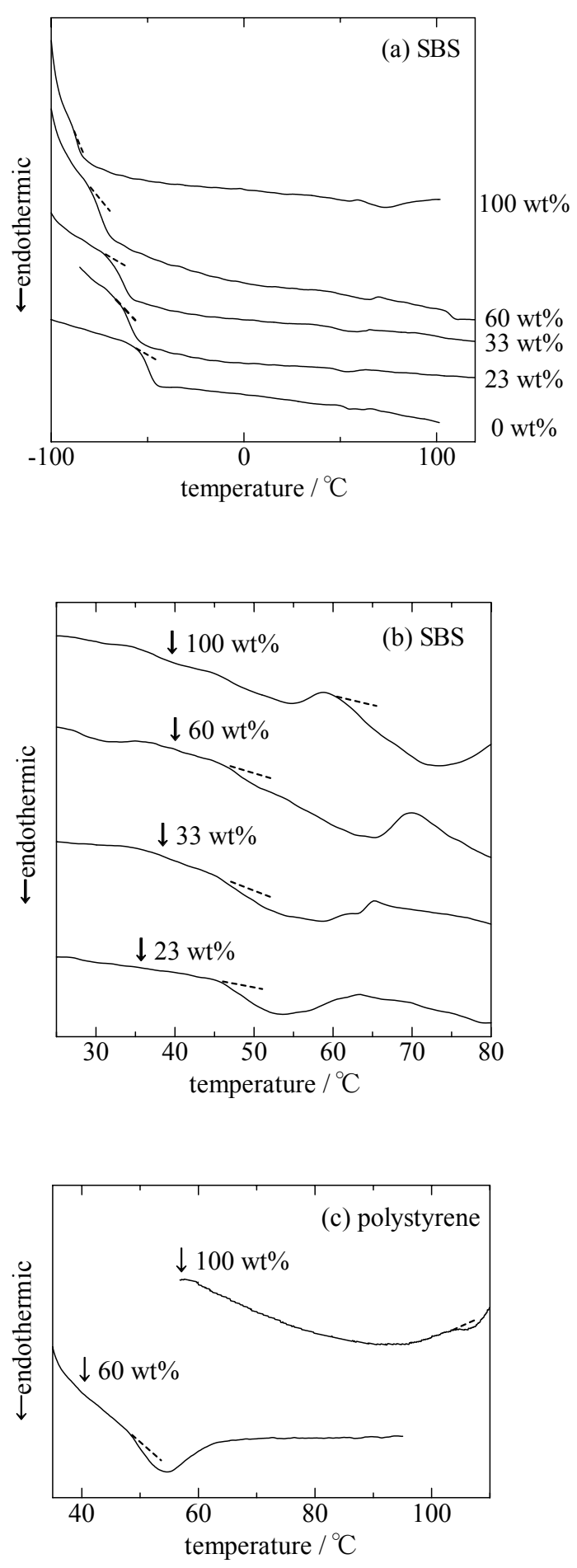

Fig. 1. The DSC curves for the gels of SBS and naphten oil with different SBS content (Fig. 1a and 1b). The DSC curves of naphten oil (0 wt\%) and bulk SBS (100 wt\%) are also illustrated in the figures. The DSC curves for bulk polystyrene and polystyrene in naphten oil are shown in Fig. 1c. 
butadiene units and naphten oil.

Watanabe et al. ${ }^{5)}$ detected the glass transition of the styrene units in the gels of SBS and $n$-tetradecane at $50 \pm 5{ }^{\circ} \mathrm{C}$, and argued that the low Tg was caused by the swelling of styrene units by $n$-tetradecane. Fig. 1c shows the DSC curves of the bulk sample of polystyrene $\left(\mathrm{Tg}=103^{\circ} \mathrm{C}\right)$ and that in naphten oil $\left(\mathrm{Tg}=49^{\circ} \mathrm{C}\right)$. This change of Tg suggests that the low $\mathrm{Tg}$ detected for SBS was caused by the swelling by naphten oil.

The Tg of the styrene units in bulk SBS shown in Fig. 1b was significantly smaller than that of bulk polystyrene. Masson et al. ${ }^{14)}$ carried out modulated DSC for the bulk SBS ( $M_{\mathrm{w}}=129,000$, the weight fraction of styrene was $30 \%$ ) and concluded that there existed three phases: a separated phase of butadiene, the two mixed phases of styrene and butadiene. The one of the two mixed phases was butadienerich phase, and the other is styrene-rich phase. The butadiene units were mostly insoluble in styrene, but the styrene-rich phase can dissolve small amount of butadiene units causing the depression of the Tg of the styrene-rich phase. They detected the $\mathrm{Tg}$ of styrene-rich phase at $68^{\circ} \mathrm{C}$, which was comparable with the Tg detected for the bulk sample of our SBS. Considering the reports of Watanabe et al. and Masson et al., we assigned the endothermic peaks shown in Fig. 1b to be the glass transition peaks of the styrene units swollen by small amount of naphten oil and the butadiene units.

Fig. 2 summarizes the Tg of the butadiene and styrene units in SBS gels with various contents of SBS. The Tg of the butadiene units increased by increasing the content of naphten oil of which Tg is higher than that of butadiene units. The change of Tg of the butadiene units suggests the high miscibility of the butadiene units and solvent oil. On the other hand, the $\mathrm{Tg}$ of styrene units $\left(45-50{ }^{\circ} \mathrm{C}\right)$ was almost independent of the SBS content. This constant value of the Tg indicates that the styrene units in SBS form aggregative phase almost immiscible with oil molecules. Fig. 2 also compares the results of DSC for SBS in naphten oil and 1-octadecene. The DSC curves of SBS in 1-octadecene were essentially the same as those of SBS in naphten oil expect for the melting peaks of 1 -octadecene at ca. $20^{\circ} \mathrm{C}$. This similarity of DSC curves insures the absence of selective adsorption of naphten oil to SBS.

In order to investigate the thermal properties of the phase separation of SBS, DSC measurements at higher temperature were conducted for SBS gel in naphten oil with various SBS content as shown in Fig. 3. An endothermic peak was observed at ca. $130{ }^{\circ} \mathrm{C}$ irrespective of the SBS content. As polystyrene is reported to have UCST-type phase diagrams with various hydrophobic solvents ${ }^{8,9,15,16)}$, this endothermic peak was assigned as the mixing heat of the styrene units in SBS and naphten oil.

Fig. 4 summarizes the dissolution enthalpy of SBS and that of homopolymer of styrene to naphten oil calculated from the area surrounded by the DSC curves shown in Fig. 3 and baselines. The dissolution enthalpy of SBS was significantly higher than that of polystyrene. The higher dissolution enthalpy implies the existence of attractive interaction among styrene units in SBS.

The viscoelastic measurements for SBS in naphten oil were carried out with varying the temperature. Fig. 5 shows the $G^{\prime}$ and $G^{\prime \prime}$ for SBS gels with different SBS contents. Depression

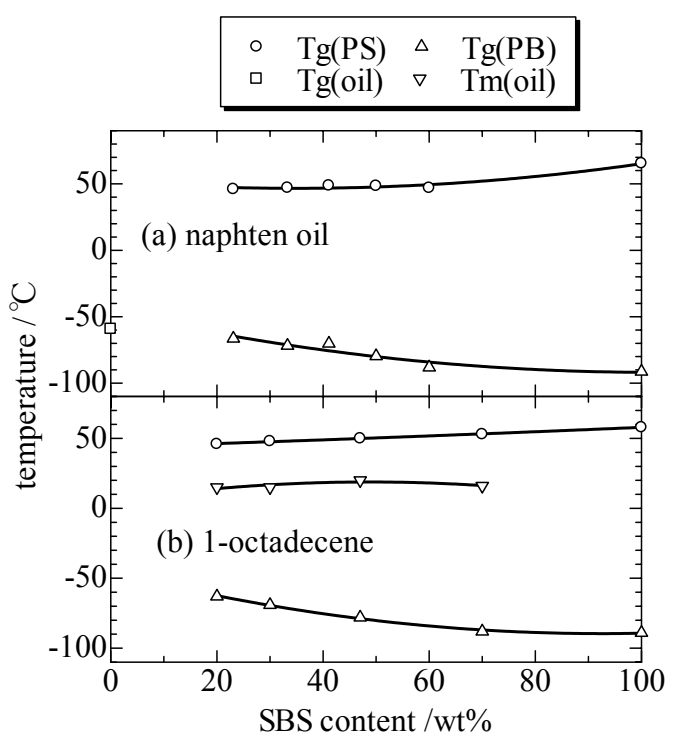

Fig. 2. The Tg of the styrene units (circles) and that of the butadiene units (triangles) of SBS in naphten oil (Fig. 2a.) and in 1-octadecene (Fig. 2b.) The Tg of naphten oil and the melting temperature $(\mathrm{Tm})$ of 1-octadecene are also indicated in this figure.

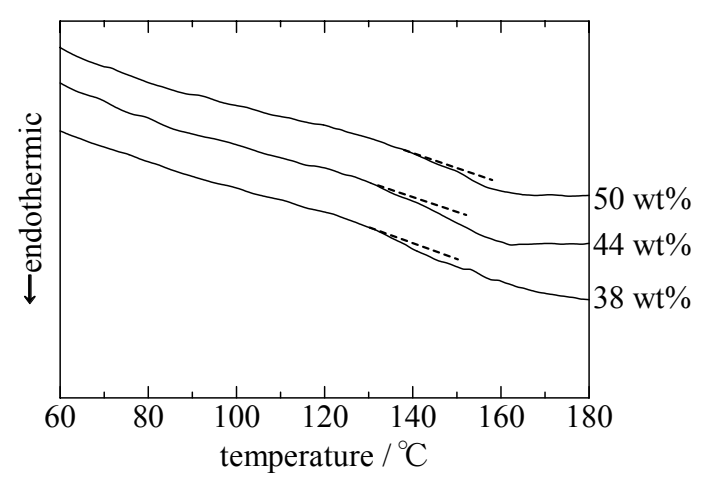

Fig. 3. DSC curves for the gels of SBS and naphten oil at high temperature. 
points of $G^{\prime}$ were observed at ca. $40{ }^{\circ} \mathrm{C}$ for all the samples (filled triangles), and $G^{\prime}$ curves had also small shoulders shifting to higher temperature by increasing SBS content (filled squares). The temperatures of depression points and shoulders were determined by plotting $\Delta \log G^{\prime} / \Delta \mathrm{T}$ against $\mathrm{T}$, where $\mathrm{T}$ stands for the temperature.

Fig. 6 is a typical plot to determine depression points and shoulder points. By increasing T, $\Delta \log G^{\prime} / \Delta \mathrm{T}$ decreases slowly below $50{ }^{\circ} \mathrm{C}$, then decreases steeply at ca.50-80 ${ }^{\circ} \mathrm{C}$. The temperature dependence of $\Delta \log G^{\prime} / \Delta \mathrm{T}$ is poor at ca.80-110 ${ }^{\circ} \mathrm{C}$, but $\Delta \log G^{\prime} / \Delta \mathrm{T}$ decreases drastically at ca.110-130 ${ }^{\circ} \mathrm{C}$. The depression point shown in a filled triangle, was determined at the temperature when $\Delta \log G^{\prime} / \Delta \mathrm{T}$ begins to decrease steeply (ca.

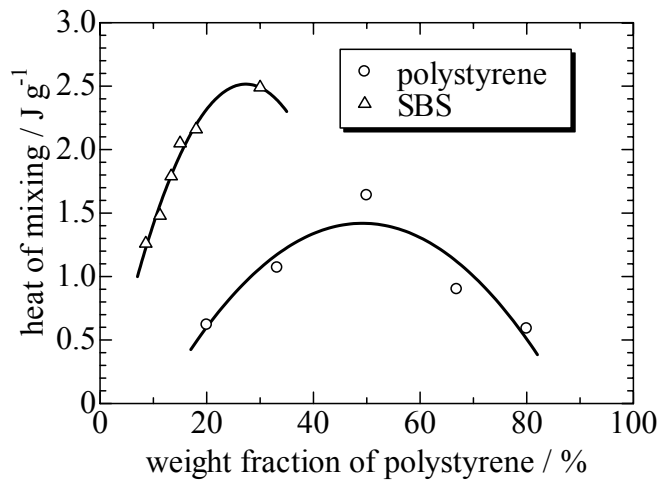

Fig. 4. The heat of mixing of SBS and naphten oil, and that of polystyrene and 1-octadecene. The solid lines are guidelines.

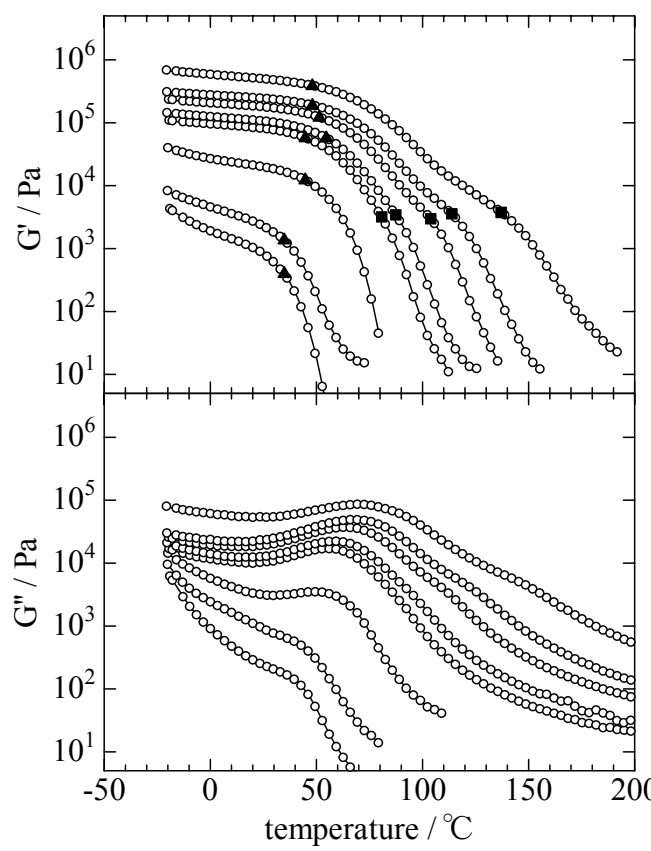

SBS

$60 \mathrm{wt} \%$ $50 \mathrm{wt} \%$ $44 \mathrm{wt} \%$ $38 \mathrm{wt} \%$ $33 \mathrm{wt} \%$ $23 \mathrm{wt} \%$ $9 \mathrm{wt} \%$ $7 \mathrm{wt} \%$

SBS $60 \mathrm{wt} \%$ $50 \mathrm{wt} \%$ $44 \mathrm{wt} \%$ $38 \mathrm{wt} \%$ $33 \mathrm{wt} \%$ $23 \mathrm{wt} \%$ $9 \mathrm{wt} \%$

$7 \mathrm{wt} \%$
Fig. 5. The results of the viscoelastic measurements for gels of SBS and naphten oil. The filled triangles and squares are depression points and shoulder temperatures described in the text.
$50{ }^{\circ} \mathrm{C}$ in Fig. 6). The shoulder point illustrated in a filled square was also determined at the temperature when $\Delta \log G^{\prime} / \Delta \mathrm{T}$ begins to decrease drastically (ca. $110^{\circ} \mathrm{C}$ in Fig. 6).

Fig. 7 summarizes specific temperatures measured by DSC, viscoelastic measurements, and tilting test tube methods for SBS in naphten oil. T1(G) and T2(G) are the temperatures of the depression points and that of the shoulders in $G^{\prime}$ curves shown in Fig. 5. T1(DSC) is the temperatures of the endothermic transitions in Fig. 1b. T2(DSC) is the temperature of endothermic peak in Fig. 3. T(flow) is the gel melting temperature determined by tilting test tube method. T1(G) well agreed with T1(DSC) assigned as the Tg of the styrene units. T2(G) was almost the same temperature as T(flow), but had no relation with T2(DSC) assigned as the UCST of the styrene units and naphten oil.

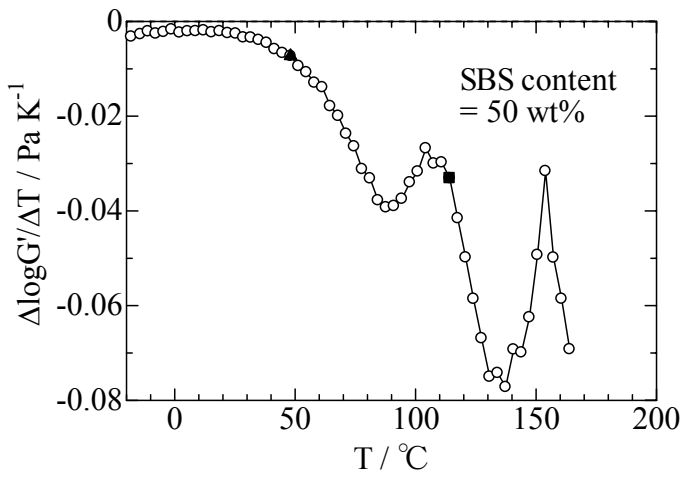

Fig. 6. A typical plot of $\Delta \log G^{\prime} / \Delta \mathrm{T}$ to determine the depression point and shoulder temperature which are designated with filled a triangle and square, respectively. The SBS content was $50 \mathrm{wt} \%$.

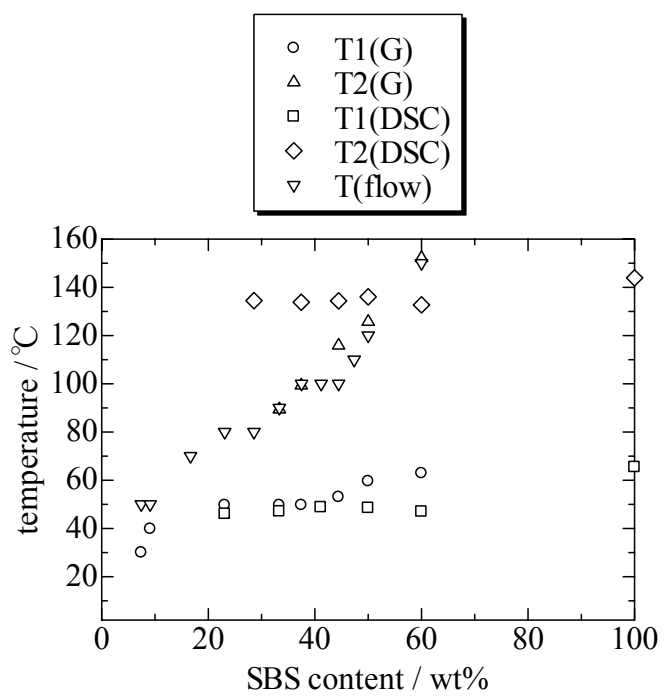

Fig. 7. Specific temperatures measured by DSC, viscoelastic measurements, and tilting test tube method for SBS in naphten oil. The meanings of the symbols are described in the text. 


\section{DISCUSSION}

The independence of Tg of the styrene units in SBS to the SBS content and the dependence of the Tg of the butadiene units shown in Fig. 2 suggest that the butadiene units form a miscible phase with naphten oil, but the styrene units were almost immiscible. The greater heat of mixing of SBS than that of polystyrene to naphten oil implies the existence of some aggregative interaction in SBS gels. These results support the microphase-separated structure proposed by Watanabe et al. ${ }^{5}$, which is constructed by the quasinetwork formed by the framework of butadiene units crosslinked by the aggregative phase of styrene units.

The mechanism of the melt of SBS gel can be investigated by the data in Fig. 7. The melting temperature, T(flow) of SBS gel was comparable with the shoulder temperature of $G^{\prime}$ curve (T2(G)) and significantly higher than the Tg of the styrene units (T1(DSC)). Watanabe et al. ${ }^{5)}$ proposed that the chain transfer process of the styrene units from one aggregative phase to another induces the change of SBS in $n$-tetradecane from rubbery state to plastic fluid. The specific temperatures in Fig. 7 show that the transition proposed by Watanabe et al. occurs at the temperature higher than Tg of the styrene units. The micro Brownian motion of the styrene units is available at the temperature slightly higher than Tg, but the aggregative interaction among the styrene units disturbs the chain transfer. By increasing the temperature, enhanced micro Brownian motion finally overcomes the aggregative interaction. At this temperature, the styrene chains can temporary dissolve into the phase of butadiene units and naphten oil, and then enter another aggregative styrene phase. This chain transfer process of the styrene units induces the flow of SBS gel.

\section{CONCLUSION}

Differential scanning calorimetry (DSC) and viscoelastic measurements were carried out for the oil gels of styrenebutadiene-styrene triblock copolymer (SBS) and naphten oil. There were three endothermic peaks assigned as the glass transition of the butadiene units, that of the styrene units, and the mixing of the styrene units and naphten oil, respectively, in the DSC curves of SBS gels. The dependence of glass transition temperature (Tg) of the butadiene units and the independence of the Tg of styrene units on the SBS content of the SBS gels suggest that the butadiene units form miscible phase with naphten oil, while the styrene units make immiscible aggregative phase.

The temperature dependence curve of $G^{\prime}$ had a depression point at the temperature near the Tg of the styrene units measured by DSC. This curve also had a shoulder at higher temperature which agreed with the gel melting temperature determined by tilting test tube method.

These results support the gel structure proposed by Watanabe et al. ${ }^{5}$, which is constructed by the quasinetwork composed of rigid styrene units connected with butadiene units. The gel began to flow at the temperature slightly higher than the Tg of the styrene units when the micro Brownian motion overcomes the aggregative interaction among the styrene units, and induce the chain transfer of styrene units.

\section{REFERENCES}

1) Takigawa T, Kashihara H, Urayama K, Masuda T, Polymer 33 (1992) 2334.

2) Hodd KA, Reader AL, British Polym J 8 (1976) 131.

3) D’Aprano A, Fuoss RM, J Polm Sci. Polym Phys Ed 7 (1969) 1101.

4) Chung CI, Griesbach HL, Young L, J Polm Sci Polym Phys Ed 18 (1980) 1237.

5) Watanabe H, Kuwahara S, Kotaka T, J Rheology 28 (1984) 393.

6) Stribeck N, Polizzi S, Zachmann HG, Rev Roumaine Chim 34 (1989) 635.

7) Boyer RF, Baer E, Hiltner A, Macromolecules 18 (1985) 427.

8) Wellinghoff S, Shaw J, Baer E, Macromolecules 12 (1979) 932.

9) Nakaoki T, Inaji Y, Polym J 34 (2002) 539.

10) Kotaka T, White JL, Trans. Soc. Rheol. 17 (1973) 587.

11) Kotaka T, Watanabe H, Nihon Reoroji Gakkaishi 10 (1982) 24.

12) Watanabe $H$, Kotaka $T$, Hashimoto $T$, Shibayama M, Kawai $H$, J Rheology 26 (1982) 153.

13) Watanabe H, Kotaka T, Polymer Eng. Rev. 4 (1984) 73.

14) Masson J-F, Bundalo-Perc S, Delgado A, J. Polym. Sci. B Polym. Phys. 43 (2005) 276.

15) Tanaka A, Kago K, Uchida Y, Nagata H, Nitta K, Polymer 42 (2001) 137.

16) Izumi Y, Kobunshi Ronbunshu, 55 (1998) 749. 